\title{
Research on Lateral Force of Pile Based on Liquefaction Site Effect
}

\author{
Zuo Xi $\mathbb{D}^{1}$ and Zhou Enquan $\mathbb{D}^{2}$ \\ ${ }^{1}$ Institute of Architectural Engineering, Jinling Institute of Technology, Nanjing 211169, China \\ ${ }^{2}$ Faculty of Civil Engineering and Mechanics, Jiangsu University, Zhenjiang 212013, China \\ Correspondence should be addressed to Zuo Xi; zuoxi82@126.com
}

Received 25 July 2019; Revised 21 October 2019; Accepted 8 November 2019; Published 23 January 2020

Academic Editor: Filippo Santucci de Magistris

Copyright (c) 2020 Zuo Xi and Zhou Enquan. This is an open access article distributed under the Creative Commons Attribution License, which permits unrestricted use, distribution, and reproduction in any medium, provided the original work is properly cited.

\begin{abstract}
This paper presents a theoretical investigation on the lateral force of pile in liquefaction site. Regarded liquefied soil as fluid, the vector method can be used to analyze the liquefaction velocity field and solve the analytical solution of the dynamic field by making use of the principle of fluid mechanics. In addition, by solving the velocity field with vector symbol operation method, the analytical expression of the lateral force in the liquefied flow field is obtained, and the sensitivity of the parameters in the analytical expression is analyzed. The results show that the stress field of the pile contains both the pressure resistance caused by surface pressure and the friction resistance caused by shear stress, when the liquefied soil flows laterally. The lateral forces on the pile are mainly composed of inertial forces and damping forces and are related to density, fluid viscosity, pile radius, and vibration frequency. With the increase of density, fluid viscosity, and pile radius, the added mass and added damping increase gradually. In a certain range, added mass and added damping are sensitive to vibration frequency.
\end{abstract}

\section{Introduction}

Sand liquefaction caused by almost every earthquake is accompanied by large deformation, and the lateral displacement caused by seismic liquefaction causes great damage to the pile foundation [1]. Hamada $[2,3]$ conducted in-depth field investigations and studied liquefaction sites and their effects in the light of the 1964 Kobe earthquake in Japan and the 1983 Chubu earthquake, in which most pile foundation damage in liquefaction sites was recorded. In 1994, the Northridge earthquake in the United States and the Hanshin earthquake in Japan in 1995 caused damage to the pile foundation of numerous bridges, buildings, storage tanks, wharves, and other structures in the liquefaction area. In addition, pile foundation damage in liquefaction sites was found among many earthquakes in Mexico, Costa Rica, India, Chile, and other countries. Cubrinovski et al. [4] reported in detail the seismic damage of bridge pile foundations in liquefaction sites in several earthquakes in New Zealand during 2010-2011. The Magnitude 9.0 earthquake that struck in the East Japan Sea on March 11, 2011, caused a large-scale liquefaction. A large number of piles on the banks of the Tone River are destroyed by site liquefaction [5]. In China, the Tangshan earthquake in 1976 caused the collapse of the pile foundation bridges such as the Yuehe Bridge, Shengli Bridge, and Luanhe Highway Bridge due to sand liquefaction and lateral displacement of the soil. In the 2008 Wenchuan earthquake, more than 10 bridges were subjected to different degrees of liquefaction damage [6].

The researchers mainly used the solid mechanics concept of pile-soil interaction to explain the failure of pile foundation in liquefiable site. It is believed that the inertial force of the upper structure is the main reason for the strength failure of the pile foundation. Ting [7] through the horizontal load test of the full-size single pile in the liquefaction site found that the soil pressure between the pile and soil under dynamic load is lower than the corresponding value under static load. Tokimatsu and Suzuki [8] used a large-scale shaking table model test to study the pore pressure response of the soil around the pile and its influence on the p-y curve. Yao et al. [9] used the shaking table model test to study the dynamic interaction behavior of pile-soil-superstructure system during liquefaction and used the centralized mass model to analyze the dynamic response. Choobbasti et al. [10] used the finite 
difference method to establish a model and studied the response of the pile-soil interaction system after soil liquefaction. Samui et al. [11] analyzed the lateral buckling of pile groups caused by the axial loads on pile foundation under the condition of soil liquefaction around the pile. $\mathrm{Li}$ and Motamed [12] established a two-dimensional nonlinear dynamic finite element model, studied the interaction of pilesoil system under the consideration of two-dimensional effective stress, and compared the analysis results of finite element model and test results. Wang et al. $[13,14]$ conducted a series of shaking table test and found that sand liquefaction will cause the foundation acceleration response to change and the maximum bending moment of the pile appears in the pile head position during the vibration process. Hussien et al. [15] carried out a series of centrifuge experiments, mainly studied the dynamic response characteristics of pile group in sand, and compared the bending moment distribution of pile foundations.

In recent years, lateral displacement caused by seismic liquefaction has caused great losses to pile. Due to the significant difference between the mechanical properties of the liquefaction soil and the solid soil, such as the low shear resistance, high fluidity, and other fluid properties, it is a novel research idea to study the liquefaction of saturated sand soil on the basis of fluid mechanics. The basic idea of adopting fluid mechanics to study the liquefaction effect is to view the liquefied sand as a kind of fluid and then to study the liquefaction effects such as flow deformation or soilstructure interaction by using the computational methods of mechanics.

Relevant scholars have done some researches and analysis and have obtained some useful research conclusions. Towhata et al. [16] took the liquefied soil as fluid for the first time in the analysis of large lateral deformation of liquefied soil. Based on the results of the shaking table test, the lateral deformation curve of liquefied soil was assumed to be the sinusoidal function curve with the maximum at the top and minimum at the bottom of the layer. After that, Hamada and Wakamatsu [17] carried out extensive surveys on the earthquakes that occurred in Japan in the 1980s and 1990s and conducted a series of shaking table tests to study the flow characteristics of liquefied soil and obtained two important conclusions: (1) Liquefied soil is highly close to a kind of pseudoplastic fluid. With the dissipation of pore water pressure after the earthquake, the stiffness of liquefied soil is recovered and gradually converted to solid. (2) The flow of liquefied soil meets the Reynolds similarity rate, and its flow velocity is proportional to the square root of the thickness of the liquefaction layer. These conclusions have played an important role in promoting the flow deformation analysis of liquefied soil, and their correctness has been proved by subsequent studies. Thereafter, Tamate and Towhata [18] regarded the liquefied soil as viscous fluid and analyzed the flow characteristic of liquefied sand by using the principle of minimum potential energy. Hadush et al. [19] treated liquefied sand as Bingham fluid with yield strength, proposed and proved a numerical method based on fluid dynamics theory to predict deformation caused by liquefied soil, and used this method to reproduce liquefaction flow by the shaking table test. Sawicki and Mierczyński [20] regarded liquefied sand as a fluid and studied the settlement of buildings in liquefied sand and the flow slip of the inclined slope by using the Navier-stokes formula, a classical equilibrium equation in fluid mechanics. Huang et al. [21] regarded liquefied sand as viscous fluid and analyzed the flow deformation of liquefied sand by using the VOF model. Monstassar and de Buhan [22] regarded liquefied sand as Bingham fluid with yield strength and developed a simplified one-dimensional numerical model to analyze the horizontal displacement of liquefied sand. Chen et al. [23] studied the hydromechanical properties of liquefied sand, and the results showed that the behavior of postliquefaction fine sand under monotonic axial compression loading can be described as the combination fluid model of a power-law timeindependent fluid and a power-law time-dependent fluid.

In this paper, the liquefied soil is regarded as fluid, and the dynamic field analysis of liquefaction site is carried out based on the principle of fluid mechanics and the vector symbol operation method, and the analytical solution of the kinetic force field is solved. After obtaining the analytical expression of lateral force of pile considering liquefaction effect, it is known that the lateral force of the liquefaction site on pile is mainly composed of inertia force and damping force and is related to fluid density, fluid viscosity, pile radius, and vibration frequency. Finally, the sensitivity analysis of the parameters in the analytical solution is carried out to obtain the sensitivity degree of different parameters.

\section{Study on Dynamic Distribution Field of Liquefaction Site}

\subsection{Analytical Solution of Velocity Field in Liquefaction Site}

2.1.1. Velocity Field Analysis Based on Vector Method. In the process of soil liquefaction, the soil changes from solid state to liquid state due to the increase of pore water pressure and the decrease of effective stress [24]. Therefore, by introducing the viewpoint of fluid mechanics, the velocity field of the liquefaction site considering the pile effect can be obtained, as shown in Figure 1.

Assume the radius of cross section of the pile is $R$. The density and viscosity of soil, when the liquefaction flow state occurs, are $\rho$ and $\eta$, respectively.

The velocity of the pile in the liquefied flow field is $v=v_{0} \cdot e^{-i \omega t}$, where $\omega$ is the vibration frequency of the pile. $v_{0}$ represents the velocity of the pile at the initial moment of soil liquefaction, which is a constant vector. Assume the liquefaction flow of soil is infinite. Thus, when its velocity is $v_{0}$, the lateral velocity field around the pile is $\mathbf{V}_{0}^{\prime}$. The flow velocity field of the liquefaction site caused by the pile movement is $\mathbf{V}_{0}$, and then $\mathbf{V}_{0}^{\prime}=\mathbf{V}_{0}+v_{0}$.

The liquefied soil can be viewed as incompressible fluid and can be obtained according to the following continuous equation:

$$
\nabla \cdot \mathbf{V}_{0}^{\prime}=\nabla \cdot\left(\mathbf{V}_{0}+v_{0}\right)=\nabla \cdot \mathbf{V}_{0}=0
$$




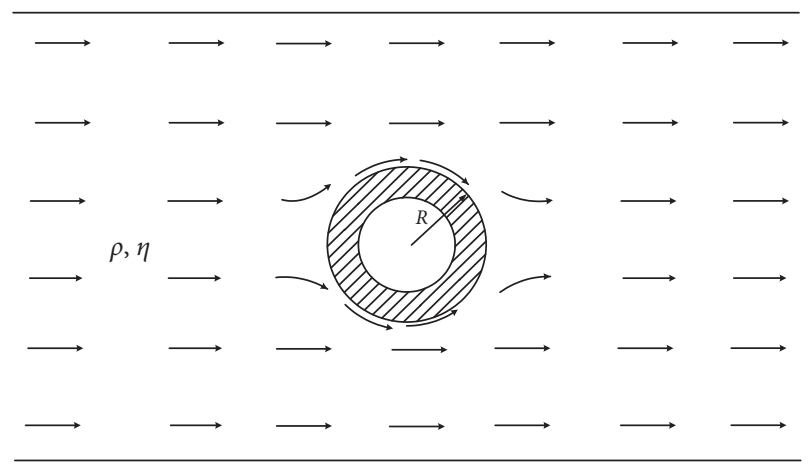

FIGURE 1: Schematic diagram of velocity field of the liquefaction site.

According to the vector theory, the polar vector $\mathbf{V}_{0}$ corresponds to the axial vector $\mathbf{A}$, to guarantee that $\nabla \cdot(\nabla \times$ A) $=0$ comes true, where $\nabla$ is the gradient, so we can obtain

$$
\mathbf{V}_{0}=\nabla \times \mathbf{A}
$$

where $\mathbf{A}$ is the axial vector, which can be expressed as the vector product of two polar vectors. Since $\mathbf{A}$ is only related to the radius $\gamma$ and velocity $v_{s}$ of the polar vector, $\mathbf{A}$ must be composed of $\gamma \times v_{0}$, and $\gamma$ can be expressed by the gradient $f^{\prime}(r) n$. For the slender structure of pile, it can be considered that $\mathbf{A}$ is independent of the axial position coordinate $z$; that is, $\mathbf{A}$ has the vector product form of $f^{\prime}(r) n \times v_{0}$. In addition, since $f^{\prime}(r) n=\nabla f$ and $v_{0}$ is a constant vector, then

$$
\mathbf{V}_{0}=\nabla \times\left(\nabla f \times v_{0}\right) \text {. }
$$

Therefore, the velocity field caused by the vibration of the pile at the velocity $v=v_{0} \cdot e^{-i \omega t}$ in the fluid field is

$$
\mathbf{V}=\left[\nabla \times\left(\nabla f \times v_{0}\right)\right] \cdot e^{-i \omega t} .
$$

\subsubsection{Solution of the Velocity Field with Vector Symbol} Operation Method. Since the liquefied soil is an incompressible fluid, the momentum equation can be obtained as follows:

$$
\frac{\partial \mathbf{V}}{\partial t}+(\mathbf{V} \cdot \nabla) \mathbf{V}=-\frac{1}{\rho} \nabla \mathbf{P}+\frac{\eta}{\rho} \Delta \mathbf{V},
$$

where $\eta$ is the fluid viscosity of liquefied soil, $\rho$ is the density of liquefied soil, and $\mathbf{P}$ is the stress field around the pile, $\Delta$ is Laplacian.

Since the fluid motion caused by soil liquefaction belongs to a uniform flow with low Reynolds number, the inertia term $(\mathbf{V} \cdot \nabla) \mathbf{V}$ in the formula can be ignored, and the linearized N-S equation can be obtained:

$$
\frac{\partial \mathbf{V}}{\partial t}=-\frac{1}{\rho} \nabla \mathbf{P}+\frac{\eta}{\rho} \Delta \mathbf{V}
$$

Take the curl from left to right of the above equation, and because $\nabla \times(\nabla \mathbf{P})=0$, we can obtain

$$
\frac{\partial(\nabla \times \mathbf{V})}{\partial t}=v_{0} \Delta(\nabla \times \mathbf{V})
$$

Substitute equation (4) into equation (7) and use the vector symbol operation to obtain

$$
\nabla\left[v_{0} \Delta^{2} f+i \omega \Delta f\right]=0 .
$$

Then

$$
v_{0} \Delta^{2} f+i \omega \Delta f=\text { Constant. }
$$

It is known from the boundary conditions at infinity that Constant $=0$; therefore,

$$
v_{0} \Delta^{2} f+i \omega \Delta f=0
$$

and the first derivative can be solved: $f^{\prime}=\mathrm{d} f$ / $\mathrm{d} r=\left(1 / r^{2}\right)\left[a e^{i k r}(r-(1 / i k)+b)\right]$, where $k=(i+1) \sqrt{\omega / 2 v_{0}}$.

Velocity field can be obtained by using the vector symbol operation method in the cylindrical coordinate system:

$$
\mathbf{V}=\left(f^{\prime \prime}-\frac{1}{r} f^{\prime}\right)\left(v_{0} \cdot n\right) n-f^{\prime \prime} v_{0} .
$$

According to the boundary condition $r=R, \mathbf{V}_{0}=v_{0}$; thus, $a=-(3 R / i k) e^{-i k R}$ and $b=-R^{3}(1-(3 / i k R)-(3 /$ $\left.k^{2} R^{2}\right)$ ).

According to equation (11), the components of $\mathbf{V}_{0}$ in the normal, tangential, and axial directions are, respectively,

$$
\begin{aligned}
& V_{0 r}=-\frac{1}{r} f v_{0} \cos \theta, \\
& V_{0 \theta}=f^{\prime \prime} v_{0} \sin \theta, \\
& V_{0 z}=0 .
\end{aligned}
$$

2.2. Analytical Solution of Stress Field in Liquefaction Site. Since flowing liquefied soil is an incompressible fluid, according to the Navier-Stokes equation and stress tensor formula, equations (3), (4), and (12)-(14) can be substituted into it to obtain

$$
\begin{aligned}
\nabla \mathbf{P}_{0} & =\eta \Delta \mathbf{V}_{0}-\left.\rho \frac{\partial \mathbf{V}}{\partial t}\right|_{t=0} \\
& =\nabla\left[\eta \Delta\left(\nabla f \cdot v_{0}\right)+i \omega \rho \nabla f \cdot v_{0}\right] .
\end{aligned}
$$

Therefore, the pressure distribution around the pile is

$$
\begin{aligned}
P_{0} & =\eta \Delta\left(\nabla f \cdot v_{0}\right)+i \omega \rho \nabla f \cdot v_{0}+P_{\infty} \\
& =\eta v_{0}\left(f^{\prime \prime \prime}+\frac{1}{r} f^{\prime \prime}-\frac{1}{r^{2}} f^{\prime}\right) \cos \theta+i \omega \rho v_{0} f^{\prime} \cos \theta+P_{\infty} .
\end{aligned}
$$

Thus, the stress field around the pile can be obtained as 


$$
\begin{aligned}
\sigma_{0 r r}^{\prime} & =2 \eta \frac{\partial V_{0 r}}{\partial r} \\
& =-2 \eta v_{0}\left[\frac{1}{r} f^{\prime \prime}-\frac{1}{r^{2}} f^{\prime}\right] \cos \theta, \\
\sigma_{0 \theta \theta}^{\prime} & =2 \eta\left[\frac{1}{r} \frac{\partial V_{0 \theta}}{\partial \theta}+\frac{V_{0 r}}{r}\right] \\
& =2 \eta v_{0}\left[\frac{1}{r} f^{\prime \prime}-\frac{1}{r^{2}} f^{\prime}\right] \cos \theta, \\
\sigma_{0 r \theta}^{\prime} & =\eta\left[\frac{1}{r} \frac{\partial V_{0 r}}{\partial \theta}+\frac{\partial V_{0 \theta}}{\partial r}-\frac{V_{0 \theta}}{r}\right] \\
& =\eta v_{0}\left[f^{\prime \prime \prime}-\frac{1}{r} f^{\prime \prime}+\frac{1}{r^{2}} f^{\prime}\right] \sin \theta . \\
\sigma_{0 \theta z}^{\prime} & =\eta\left[\frac{\partial V_{0 \theta}}{\partial z}+\frac{1}{r} \frac{\partial V_{0 z}}{\partial \theta}\right]=0, \\
\sigma_{0 z z}^{\prime} & =2 \eta \frac{\partial V_{0 z}}{\partial z}=0 . \\
\sigma_{0 z r}^{\prime} & =\eta\left[\frac{\partial V_{0 z}}{\partial r}+\frac{\partial V_{0 r}}{\partial z}\right]=0,
\end{aligned}
$$

According to the calculation results, $\sigma_{0 r r}^{\prime}, \sigma_{0 \theta \theta}^{\prime}$, and $\sigma_{0 r \theta}^{\prime}$ are nonzero values. It can be found that the stress field around the pile includes both the pressure resistance caused by the surface pressure and the friction resistance caused by the shear stress.

\section{Research Studies on Lateral Force of Pile under Liquefaction Site Flow Condition}

3.1. Analytical Solution of Lateral Force of Pile. The lateral force applied to the pile is parallel to the velocity of soil liquefaction flow. Therefore, all surface forces acting on the surface of the pile are projected to the direction of fluid velocity, and integrate along the whole cylinder of the pile. The lateral force acting on the unit length pile is

$$
\begin{aligned}
F= & \frac{1}{L} \iint_{A}\left(P_{0} \cos \theta-\sigma_{0 r r}^{\prime} \cos \theta+\sigma_{0 \theta \theta}^{\prime} \sin \theta\right. \\
& \left.+\sigma_{0 r \theta}^{\prime} \sin \theta\right) \cdot e^{-i \omega t} \mathrm{~d} A .
\end{aligned}
$$

On the pile cylinder $r=R$, according to formula (16)-(19),

$$
\begin{aligned}
P_{0} & =\left[3 \eta\left(\frac{1}{R}-i k\right)-i \omega \rho R\right] v_{0} \cos \theta+P_{\infty}, \\
\sigma_{0 r r}^{\prime} & =0 \\
\sigma_{0 \theta \theta}^{\prime} & =0 \\
\sigma_{0 r \theta}^{\prime} & =0 \\
\sigma_{0 r \theta}^{\prime} & =3 \eta v_{0}\left(\frac{1}{R}-i k\right) \sin \theta .
\end{aligned}
$$

According to formula (23) and according to the definition of fluid mechanics principle $\delta=\sqrt{2 v / \omega}$ and $v=\eta / \rho$, by integration,

$$
F=6 \pi R \sqrt{\frac{\eta \rho}{2 \omega}}\left(1+\frac{R}{3 \delta}\right) \frac{\mathrm{d} v}{\mathrm{~d} t}+6 \pi \eta\left(1+\frac{R}{\delta}\right) v,
$$

where $\eta$ is the fluid viscosity of liquefied soil, $\rho$ is the density of liquefied soil, $R$ is the radius of pile, and $\omega$ is the vibration frequency of pile.

Formula (25) shows that the lateral force of the liquefaction site on pile is mainly composed of inertia force and damping force, and its dynamic characteristics are related to density, fluid viscosity, pile radius, and vibration frequency of pile. Suppose

$$
\begin{aligned}
m_{a} & =6 \pi R \sqrt{\frac{\eta \rho}{2 \omega}}\left(1+\frac{R}{3 \delta}\right), \\
c_{a} & =6 \pi \eta\left(1+\frac{R}{\delta}\right),
\end{aligned}
$$

where $m_{a}$ is the dynamically added mass of pile and $c_{a}$ is the dynamic added damping of pile. Both of these factors reflect the dynamic characteristics of pile under transverse vibration in the liquefaction flow field.

Finally, the lateral force of liquefaction site on the pile can be expressed as

$$
F=m_{a} \dot{v}+c_{a} v
$$

\subsection{Parametric Sensitivity Analysis of Analytical Solutions of Lateral Force}

3.2.1. Method of Sensitivity Analysis. Sensitivity is defined as the derivative of a function with respect to a parameter; at the parameter $x$, sensitivity of dynamic added mass function $M(X)$ or dynamic added damping function $C(X)$ with respect to $x_{i}$ is

$$
\begin{array}{r}
S_{x_{i}}(X)=\frac{\partial M(X)}{\partial x_{i}}, \\
\text { or } S_{x_{i}}(X)=\frac{\partial C(X)}{\partial x_{i}}
\end{array}
$$

where $S_{x_{i}}$ reflects the monotonicity of function $M(X)$ or $C(X)$ on parameter $x_{i}$. The value of $S_{x_{i}}$ indicates the sensitivity of each function to the parameter $x_{i}$. The larger the value, the more sensitive the function is to $x_{i}$.

The sensitivity of density, fluid viscosity, pile radius, and vibration frequency is analyzed below, and take the reference value of each parameter as density of liquefied soil $\rho=1600 \mathrm{~kg} / \mathrm{m}^{3}$, fluid viscosity $\eta=10^{3} \mathrm{~Pa} \cdot \mathrm{s}$, radius of pile $R=0.5 \mathrm{~m}$, and vibration frequency of pile $\omega=2 \mathrm{~Hz}$. During the sensitivity analysis of each parameter, the other parameters remain unchanged based on the change of a single parameter.

3.2.2. Density of Liquefied Soil. The curves of the added mass and added damping with the density of liquefied soil are 


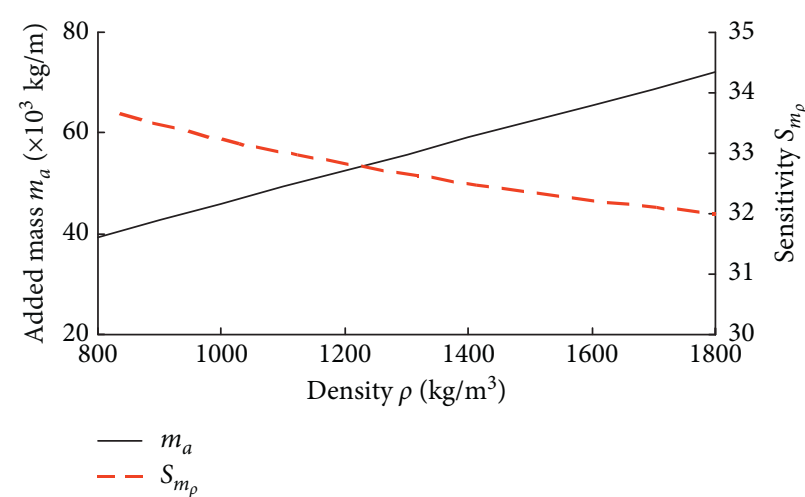

(a)

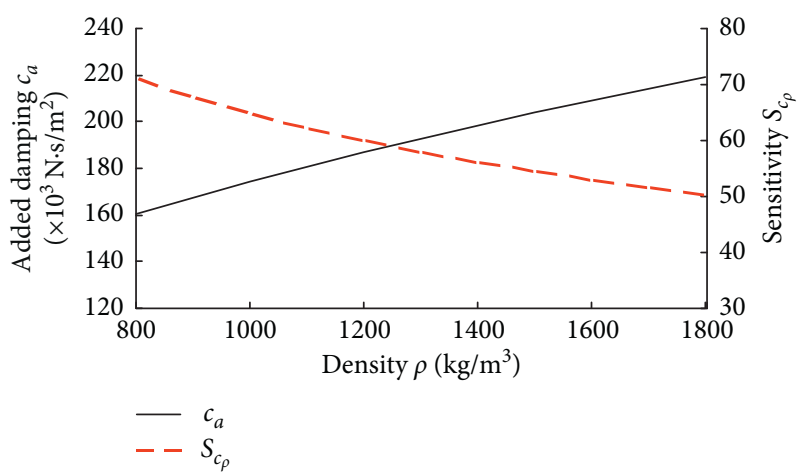

(b)

Figure 2: Influence of density on the added mass (a) and added damping (b).

shown in Figure 2. The added mass and added damping increase with fluid density, and it indicates that with the increase of fluid density, the lateral force of liquefied soil on pile increases gradually. The sensitivity decreases with the increase of density, indicating that fluid will become more sensitive to lateral force when the density of fluid becomes smaller. The variation range of sensitivity corresponding to added damping is obviously larger than that corresponding to added mass, which proves that the influence of fluid density on added damping is greater.

3.2.3. Fluid Viscosity of Liquefied Soil. The curves of added mass and added damping with the viscosity of liquefied soil are shown in Figure 3. The added mass and added damping increase with the increase of fluid viscosity, and the sensitivity decreases with the increase of fluid viscosity. It implies that the smaller the fluid viscosity, the more sensitive it is to lateral forces. And when the viscosity is between $1000 \mathrm{~Pa} \cdot \mathrm{s}$ and $2000 \mathrm{~Pa} \cdot \mathrm{s}$, and the corresponding sensitivity drops sharply. This indicates that the change of fluid viscosity has a great influence on the lateral force. The variation range of sensitivity of added damping is larger, which indicates that the influence of fluid viscosity on added damping is greater.

3.2.4. Pile Radius. The curves of added mass and added damping with pile radius are shown in Figure 4 . It can be found that as the pile radius increases, the added mass and added damping increase gradually. The sensitivity corresponding to added mass increases accordingly, while the sensitivity corresponding to added damping remains unchanged, indicating that the pile radius is sensitive to added mass, but not to added damping.

3.2.5. Vibration Frequency of Pile. The curves of the added mass and added damping with the vibration frequency of the pile are shown in Figure 5. With the increase of vibration frequency, the added mass decreases sharply and tends to be stable gradually. The added damping increases gradually, and the sensitivity corresponding to the added mass and added damping decreases rapidly at the low frequency stage and tends to a smaller value, indicating that when the frequency is less than $1 \mathrm{~Hz}$, the vibration frequency is particularly sensitive to the lateral force.

3.3. Comparative Analysis of Analytical and Numerical Solution of the Distribution Field around the Pile. Using the CFD fluid module of ABAQUS finite element platform, Abaqus/CFD provides advanced computational fluid dynamics capabilities with extensive support for preprocessing and postprocessing provided in Abaqus/CAE. These scalable parallel CFD simulation capabilities address a broad range of nonlinear coupled fluid-structural problems. Abaqus/CFD can solve the following types of incompressible flow problems: the internal or external flows that are steady-state or transient, span a broad Reynolds number range, and involve complex geometry that may be simulated with Abaqus/CFD. This includes flow problems induced by spatially varying distributed body forces.

A fluid finite element model is established to carry out numerical calculation of liquefied dynamic field, based on the principle of minimum potential energy and basic control equation of flow. A site is adopted to simulate the flow effect of the liquefaction site. The schematic diagram of the site is shown in Figure 6.

For the boundary conditions, in order to better simulate the infinite dynamic flow field, in the whole liquefied soil flow area, all the boundaries except the fluid outlet are set as the velocity inlet. Some parameters used in numerical calculation are as follows: the CFD model is adopted for liquefied soil mass, and thus, $\rho=1600 \mathrm{~kg} / \mathrm{m}^{3}$ (the soil density) and $\eta=1000 \mathrm{~Pa} \cdot \mathrm{s}$ (dynamic viscosity). The elastic model is adopted for pile, and thus, $\rho=2500 \mathrm{~kg} / \mathrm{m}^{3}$ (concrete pile density), $E=3 \times 10^{4} \mathrm{MPa} \cdot \mathrm{s}$ (elasticity modulus), and $\lambda=$ 0.35 (Poisson's ratio). In the calculation, the initial stress setting and balance of the soil are firstly carried out to obtain the stress distribution in the preliquefaction state, and then the liquefied soil is set as the fluid material for the dynamic field calculation.

The comparison of the analytical and numerical solutions of the stress distribution field around the pile is 


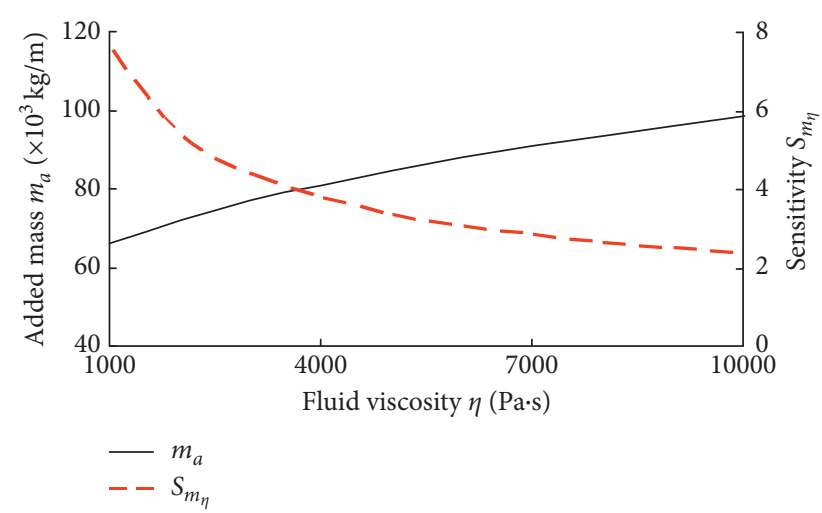

(a)

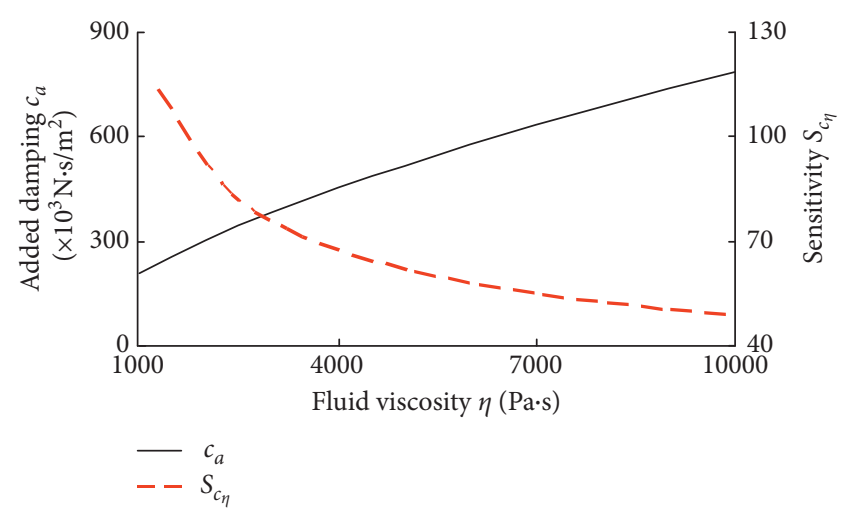

(b)

FIGURE 3: Influence of fluid viscosity on the added mass (a) and added damping (b).

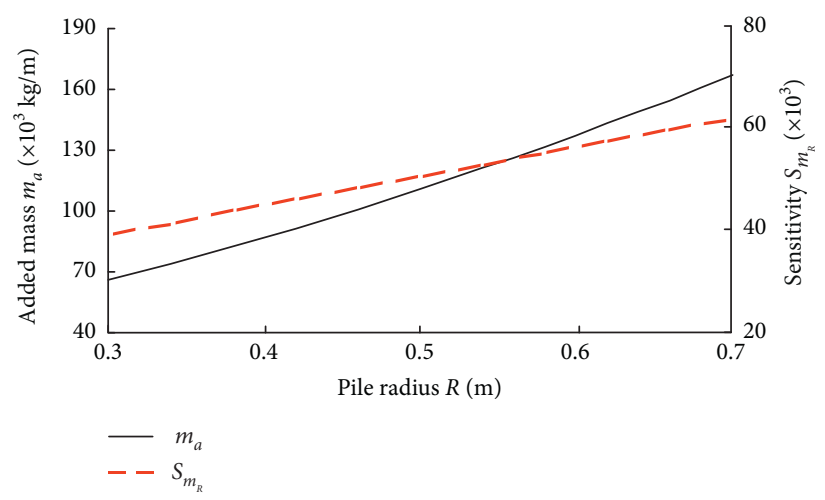

(a)

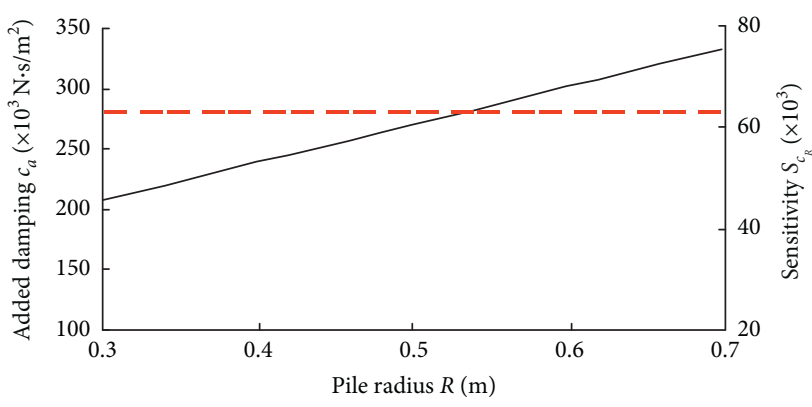

$-c_{a}$

(b)

Figure 4: Influence of pile on the added mass (a) and added damping (b).

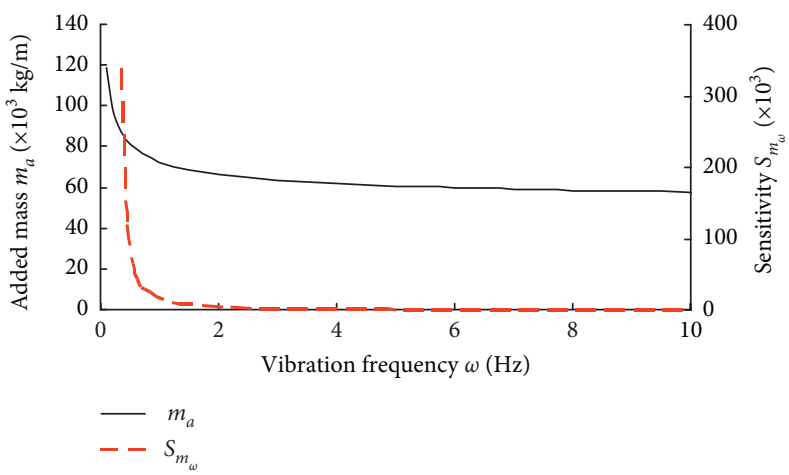

(a)

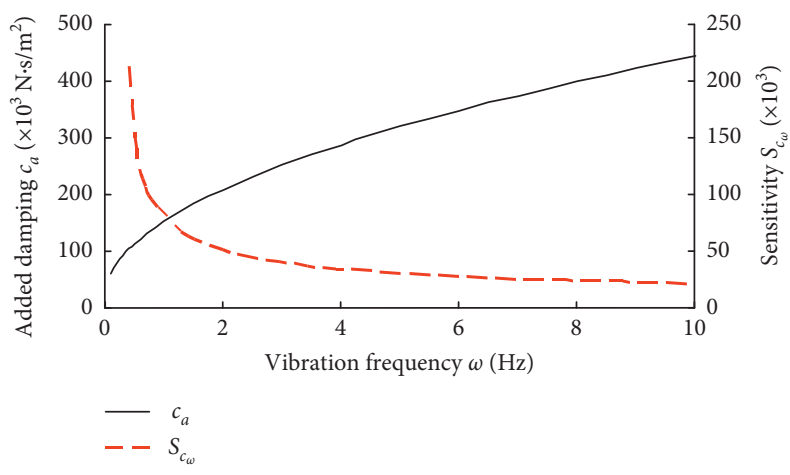

(b)

FIGURE 5: Influence of vibration frequency on the added mass (a) and added damping (b).

shown in Figure 7. It can be seen that the distribution trend of the analytical solution of the stress field is basically the same as that of the finite element solution around the pile and the stress on the pile structure is mainly distributed in the head-on position of liquefied soil flow. This result is consistent with the stresses around the pile in the process of seabed consolidation movement in Literature [25]. 


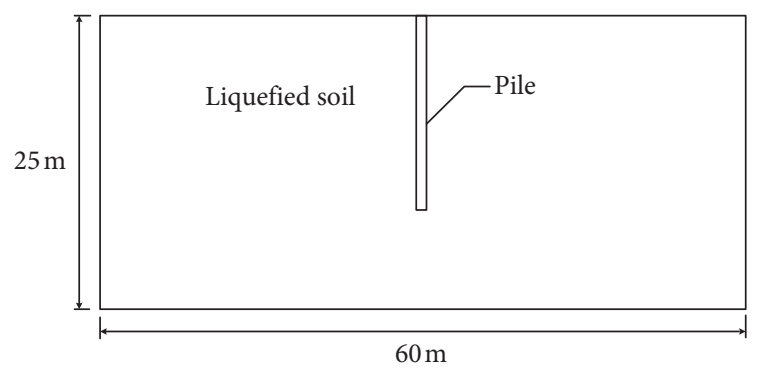

(a)

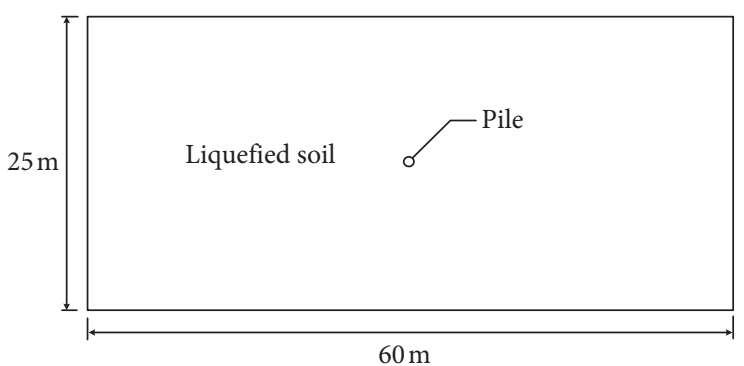

(b)

Figure 6: Schematic diagram of the liquefied site. (a) Sectional view. (b) Vertical view.

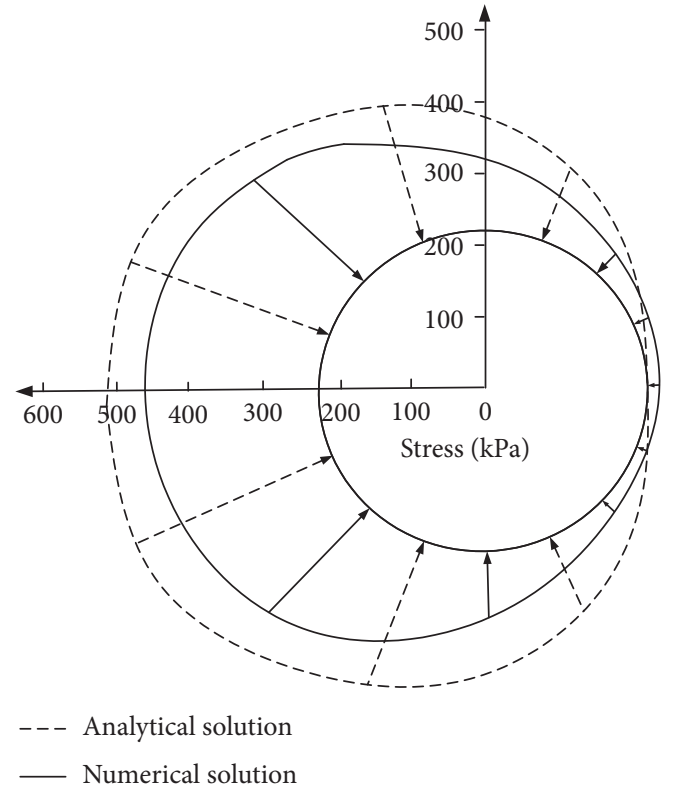

FIGURE 7: Analytical solution and numerical solution of the stress field around the pile (the section at $10 \mathrm{~m}$ depth).

\section{Conclusion}

Regarding the liquefied soil as fluid, the dynamic field analysis of liquefaction site is carried out based on the principle of fluid mechanics and the vector symbol operation method, and the analytical solution of the kinetic force field is solved. The analytical expression of the lateral force of pile is obtained considering the liquefaction effect, the sensitivity analysis of the parameters in the analytical solution is carried out, and then the influence of different parameters on the lateral force of pile is obtained. The main conclusions are as follows:

(1) According to the analytical solution calculated by the liquefaction dynamic field theory, it can be known that when the liquefied soil flows laterally, the stress field around the pile includes the pressure resistance caused by the surface pressure and the friction resistance caused by the shear stress.

(2) The lateral force of liquefied soil on pile is mainly composed of added inertia and added damping, which are related to density, fluid viscosity, pile radius, and vibration frequency.

(3) With the increase of density, fluid viscosity, and pile radius, the added mass and added damping are gradually increasing. The influence of density and fluid viscosity on the added damping is greater, and the pile radius is only sensitive to the additional mass. Vibration frequency is particularly sensitive to added mass and added damping within a certain range.

\section{Data Availability}

The data used to support the findings of this study are available from the corresponding author upon request.

\section{Conflicts of Interest}

The authors declare that there are no conflicts of interest regarding the publication of this paper.

\section{Acknowledgments}

The authors gratefully acknowledge the financial support for this study from the project of the National Natural Science Foundation of China (Grant no. 51408281), the Natural Science Foundation of Jiangsu Province (Grant no. BK20140108), and Qing Lan Project (2016).

\section{References}

[1] H. S. Liu, F. P. Xu, P. C. Li et al., "Lateral spread and large ground displacement caused by earthquake liquefaction," Special Structures, vol. 14, no. 2, pp. 47-50, 1997.

[2] M. Hamada, "Large ground deformations and their effects on lifelines: 1964 Niigata earthquake: case studies of liquefaction and lifelines performance during past earthquake," Technical Report NCEER-92-0001, Japanese Case Studies, National Centre for Earthquake Engineering Research, Buffalo, NY, USA, 1992.

[3] M. Hamada, "Large ground deformations and their effects on lifelines: 1983 Nihonkai-Chubu earthquake, case studies of liquefaction and lifelines performance during past earthquake," Technical Report NCEER-92-0001, Japanese Case Studies, National Centre for Earthquake Engineering Research, Buffalo, NY, USA, 1992. 
[4] M. Cubrinovski, J. Haskell, A. Winkley, K. Robinson, and L. Wotherspoon, "Performance of bridges in liquefied deposits during the 2010-2011 Christchurch (New Zealand) earthquakes," Journal of Performance of Constructed Facilities, vol. 28, no. 1, pp. 24-39, 2014.

[5] D. Pradel, J. Wartman, and B. Tiwari, "Impact of anthropogenic changes on liquefaction along the Tone River during the 2011 Tohoku earthquake," Natural Hazards Review, vol. 15, no. 1, pp. 13-26, 2014.

[6] Z. H. Wu and Z. C. Zhang, "Types and examples of geological hazards in Wenchuan Ms 8.0 earthquake," Acta Geologica Sinica, vol. 82, no. 12, pp. 1747-1757, 2008.

[7] J. M. Ting, "Full-scale cyclic dynamic lateral pile responses," Journal of Geotechnical Engineering, vol. 113, no. 1, pp. 30-45, 1987.

[8] K. Tokimatsu and H. Suzuki, "Power pressure response around pile and its effects on p-y behvaior during soil liquefaction," Soils and Foundations, vol. 44, no. 6, pp. 101-110, 2004.

[9] S. Yao, K. Kobayashi, N. Yoshida, and H. Matsuo, "Interactive behavior of soil-pile-superstructure system in transient state to liquefaction by means of large shake table tests," Soil Dynamics and Earthquake Engineering, vol. 24, no. 5, pp. 397-409, 2004

[10] A. J. Choobbasti, M. Saadati, and H. R. Tavakoli, "Seismic response of pile foundations in liquefiable soil: parametric study," Arabian Journal of Geosciences, vol. 5, no. 6, pp. 1307-1315, 2012.

[11] P. Samui, S. Bhattacharya, and T. G. Sitharam, "Support vector classifiers for prediction of pile foundation performance in liquefied ground during earthquakes," International Journal of Geotechnical Earthquake Engineering, vol. 3, no. 2, pp. 42-59, 2012.

[12] G. Li and R. Motamed, "Finite element modeling of soil-pile response subjected to liquefaction-induced lateral spreading in a large-scale shake table experiment," Soil Dynamics and Earthquake Engineering, vol. 92, pp. 573-584, 2017.

[13] R. Wang, J. M. Zhang, and G. Zhang, "Centrifuge shaking table test on single pile lateral spreading soil," Engineering Mechanics, vol. 29, no. 10, pp. 98-105, 2012.

[14] R. Wang, X. Liu, and J.-M. Zhang, "Numerical analysis of the seismic inertial and kinematic effects on pile bending moment in liquefiable soils," Acta Geotechnica, vol. 12, no. 4, pp. 773-791, 2016.

[15] M. N. Hussien, T. Tobita, S. Iai, and M. Karray, "Soil-pilestructure kinematic and inertial interaction observed in geotechnical centrifuge experiments," Soil Dynamics and Earthquake Engineering, vol. 89, no. 10, pp. 75-84, 2016.

[16] I. Towhata, Y. Sasaki, K. Tokida, H. Matsumoto, Y. Tamari, and K. Yamada, "Prediction of permanent displacement of liquefied ground by means of minimum energy principle," Soils and Foundations, vol. 32, no. 3, pp. 97-116, 1992.

[17] M. Hamada and K. Wakamatsu, "A study on ground displacement caused by soil liquefaction," Doboku Gakkai Ronbunshu, vol. 43, no. 596, pp. 189-208, 1998.

[18] S. Tamate and I. Towhata, "Numerical simulation of ground flow caused by seismic liquefaction," Soil Dynamics and Earthquake Engineering, vol. 18, no. 7, pp. 473-485, 1999.

[19] S. Hadush, A. Yashima, R. Uzuoka, S. Moriguchi, and K. Sawada, "Liquefaction induced lateral spread analysis using the CIP method," Computers and Geotechnics, vol. 28, no. 8, pp. 549-574, 2001.

[20] A. Sawicki and J. Mierczyński, "On the behaviour of liquefied soil," Computers and Geotechnics, vol. 36, no. 4, pp. 531-536, 2009.
[21] Y. Huang, W. Mao, H. Zheng, and G. Li, "Computational fluid dynamics modeling of post-liquefaction soil flow using the volume of fluid method," Bulletin of Engineering Geology and the Environment, vol. 71, no. 2, pp. 359-366, 2012.

[22] S. Montassar and P. de Buhan, "Numerical prediction of liquefied ground characteristics from back-analysis of lateral spreading centrifuge experiments," Computers and Geotechnics, vol. 52, no. 32, pp. 7-15, 2013.

[23] G. X. Chen, E. Q. Zhou, Z. H. Wang et al., "Experimental study on fluid characteristics of medium dense saturated fine sand in pre- and post-liquefaction," Bulletin of Earthquake Engineering, vol. 14, no. 8, pp. 2185-2212, 2016.

[24] Z. H. Wang, E. Q. Zhou, G. X. Chen et al., "Characteristics of solid-liquid phase change of saturated sand under cyclic loading," Chinese Journal of Geotechnical Engineering, vol. 34, no. 9, pp. 1604-1610, 2012.

[25] T. Sui, J. Zheng, C. Zhang et al., "Consolidation of unsaturated seabed around an inserted pile foundation and its effects on the wave-induced momentary liquefaction," Ocean Engineering, vol. 131, pp. 308-321, 2017. 